\title{
CONDICIONAMENTO CARDIORRESPIRATÓRIO E MARCADORES BIOQUÍMICOS DE RISCO CORONARIANO
}

\author{
Cardliorrespiratory fitmess and coronary risk markers \\ José Mário de Souza-e-Sá Júnior ${ }^{1}$, Marcos Alexandre Monteiro-Gomes ${ }^{1}$, \\ Carla Cristina Pimenta Alcaraz', José Carlos Meireles de Sousa ${ }^{1}$, \\ Francisco Harrisson de Souza ${ }^{1}$, Cynthia Torres França da Silva ${ }^{1}$, \\ Marcos de Sá Rego Fortes ${ }^{2}$, Eduardo Camillo Martinez ${ }^{2,3}$
}

\section{RESUMO}

O condicionamento cardiorrespiratório $\left(\mathrm{VO}_{2 \max }\right)$ tem se mostrado associado ao risco de doenças cardiovasculares pela literatura científica. Este estudo, de corte transversal, teve por objetivo descrever as características de $\mathrm{VO}_{2 \max }$ em sujeitos com IMC $>28 \mathrm{~kg} \cdot \mathrm{m}^{-2}$ e avaliar relações com indicadores de risco cardiovascular. A amostra foi constituída por 19 militares, da ativa, com Idade de $38,1 \pm 7,8$ anos, Massa Corporal de $89,3 \pm 7,1 \mathrm{~kg}$, Estatura de $172,7 \pm 5,6 \mathrm{~cm}$ e Percentual de Gordura Corporal (\%G) de $24,9 \pm 4,2$, este último pelo método da Pesagem Hidrostática. $\mathrm{O} \mathrm{VO}_{2 \max }$ foi predito utilizando o teste de Cooper de 12 minutos $\left(43,2 \pm 8,8 \mathrm{ml}_{\mathrm{kg}}{ }^{-1} \cdot \mathrm{min}^{-1}\right)$. Foram dosados os níveis séricos de colesterol total (CT), HDL-c, LDL-c, triglicerídeos e glicemia de jejum. O estabelecimento da associação entre as variáveis foi feito pelo coeficiente de correlação de Spearman e, para a análise dos dados de maneira categorizada, utilizou-se o teste exato de Fisher $(p<0,05)$. Os resultados mostraram correlação inversa entre $\mathrm{VO}_{2 \max }$ e CT $(r=-0,490)$, LDL-c $(r=-0,539)$ e direta com o HDL-c $(r=0,591) . O V_{2 m a x}$, acima de $37,1 \mathrm{ml} \mathrm{kg}^{-1} \cdot \mathrm{min}^{-1}$, relacionou-se com a diminuição da prevalência dos níveis séricos de risco de LDL-C e CT, com a redução da prevalência de sujeitos com $\% \mathrm{G}>25 \%$ e com o aumento da prevalência de indivíduos com HDL-c>40mg.dl ${ }^{-1}$.

Palavras-chave: Doenças Cardiovasculares, Militares, Nível de Atividade Física, Fatores de Risco

\section{ABSTRACT}

The low cardiorrespiratory fitness was associated with the coronary risk factors and the mortality by heart diseases. The aim of this correlational-descriptive and transversal study was to investigate the association between the aerobic capacity with coronary risk in nineteen

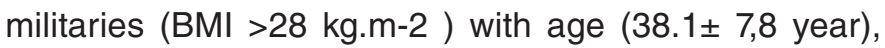
weight $(89.3 \pm 7.1 \mathrm{~kg})$ and height $(172.7 \pm 5.6 \mathrm{~cm})$. The BMI $(89.3 \pm 7.1)$ was calculated, as well as body fat percentual $(\% \mathrm{BF})$ by hydrostatic weight $(24.9 \pm 4.2)$. The $\mathrm{VO}_{2 \max }$ assessment based on the Cooper Test was (43.2 $\pm 8.8 \mathrm{ml} . \mathrm{kg}-1 . \mathrm{min}-1)$. The plasma concentrations of total cholesterol (TC), HDL-c, LDL-c, triglycerides and glycemia were dosed in fasting state. The Spearman Correlation analysis was used to examine the association between the variables and Fisher's exact test was used for the analyzes categorized of the data $(p<0.05)$. The results had shown inverse correlation between $\mathrm{VO}_{2 \max }$ and $\mathrm{TC}(\mathrm{r}=-0.490)$, and directly with HDL-C $(r=0.591)$. The $\mathrm{VO}_{2 \max }$, over $37.1 \mathrm{ml} . \mathrm{kg}-1 . \mathrm{min}-1$, became related with low prevalence of risk serum level of LDL-c e TC, \%BF>25\% and with the increase of prevalence in individual with HDL-c > 40mg.dl-1.

Key words: Cardiovascular Disease, Military, Physical Activity, $\mathrm{VO}_{2 \max }$

1. Escola de Educação Física do Exército (EsEFEx) - Rio de Janeiro - RJ - Brasil.

2. Instituto de Pesquisa da Capacitação Física do Exército (IPCFEx) - Rio de Janeiro - RJ - Brasil.

3. Escola Nacional de Saúde Pública Sergio Arouca/Fundação Oswaldo Cruz - Rio de Janeiro - RJ - Brasil.

Revista de Educação Física 2008;143:41-46. Rio de Janeiro (RJ) - Brasil.

Recebido em 15.07.2008. Aceito em 28.10.2008. 


\section{INTRODUÇÃO}

Embora a mortalidade por doenças cardiovasculares (DCV) venha declinando, no hemisfério norte, sua prevalência permanece como a maior causa de morte desde os anos 90 . Em 2001, 43,6\% dos gastos com internações de alta complexidade, no Brasil, estiveram relacionados com complicações cardiológicas. Além disso, excetuando-se as causas relacionadas à gravidez e ao parto, as doenças cardiorrespiratórias foram responsáveis, respectivamente, por $43,5 \%$ e $33,5 \%$ das taxas de mortalidade e morbidade nacional ${ }^{1,2}$.

A cardiologia foi responsável por $12,8 \%$ do total dos 873.973 atendimentos ambulatoriais e $36 \%$ da geração de exames complementares - excluindo-se os exames radiológicos simples - realizados, em 2004, nas organizações militares de saúde do Exército Brasileiro $(\mathrm{EB})^{3}$.

$\mathrm{O}$ consumo máximo de oxigênio $\left(\mathrm{VO}_{2 \max }\right)$ reflete a função cardíaca, circulatória e respiratória do organismo $0^{4,5}$. Laukkanen et al. ${ }^{6}$ sugeriram que o $\mathrm{VO}_{2 \max }$ poderia ser considerado como fator independente de predição de mortalidade por doença coronariana, após constatar que homens que possuíam $\mathrm{VO}_{2 \max }$ menor que 27,6 $\mathrm{ml} . \mathrm{kg}^{-1} \cdot \mathrm{min}^{-1}$ mostraram 3,09 vezes maior risco de morte do que aqueles que possuíam $37,1 \mathrm{ml} \cdot \mathrm{kg}^{-1} \cdot \mathrm{min}^{-1}$.

Este estudo descritivo-correlacional, de corte transversal, teve por objetivo avaliar suas possíveis relações do $\mathrm{VO}_{2 \max }$ com os níveis plasmáticos de indicadores de risco coronariano - colesterol total (CT), LDL-colesterol (LDL-C), HDL-colesterol (HDL-c), triglicerídeos (TG) e glicemia de jejum - em sujeitos com sobrepeso e obesidade.

\section{METODOLOGIA}

Foram selecionados 19 militares, da ativa, todos voluntários e servindo no Forte São João, na Urca/RJ, com IMC maior ou igual a $28 \mathrm{~kg} \cdot \mathrm{m}^{-2}$, sem limitação física para realizar teste de esforço em esteira. A amostra apresentou idade de $38,1 \pm 7,8$ anos (média \pm desvio-padrão), massa corporal de $89,3 \pm 7,1 \mathrm{~kg}$, estatura de $172,7 \pm 5,6 \mathrm{~cm} \mathrm{e}$ $24,9 \pm 4,2 \%$ de gordura corporal (\%G) (TABELA 1).

Para a seleção da amostra, após escolha aleatória dentre os que possuíam o perfil desejado, os militares foram contatados, individual e reservadamente, em seus locais de serviço, sendo convidados a participar do estudo.
Após estarem esclarecidos quanto a eventuais dúvidas e atestarem a vontade de fazer parte da amostra, assinaram o termo de consentimento livre e esclarecido e tiveram seus exames agendados.

A massa corporal foi medida no primeiro horário da manhã, em jejum, utilizando-se balança eletrônica digital Filizola, com precisão de 50 gramas e capacidade para 150 quilos, com o sujeito descalço, trajando somente sunga, no centro da balança, de frente para o avaliador e de costas para o display de resultado. Após isto, a estatura foi mensurada em estadiômetro de parede fixo, com precisão de 1 milímetro. Esta medida foi tomada do chão até o vértex da cabeça, estando o avaliado completamente ereto com a coluna cervical em posição neutra.

Foi aplicado um questionário auto-respondido, com questões de histórico individual de doenças, rotinas gerais, atividade física e uso de medicamentos. Ao avaliado, foi facultada a opção de deixar de responder qualquer pergunta.

Para a predição do condicionamento cardiorrespiratório, utilizou-se o teste de Cooper ${ }^{7}$ (1968) de 12 minutos, por sua praticidade e pela alta correlação dos seus resultados $(\mathrm{R}=0,891)$ com $\circ \mathrm{VO}_{2 \max }$, tendo sido realizado sempre no horário entre 07:30 e 09:00 horas, em pista confeccionada em terreno plano, com demarcação a cada 50 metros, adotando-se, como resultado, a última marca ultrapassada pelo sujeito antes do término do tempo estabelecido conduta que proporcionou precisão de $1,1 \mathrm{ml} \cdot \mathrm{kg}^{-1} \cdot \mathrm{min}^{-1}$ na medida.

Considerando-se que os dados foram coletados no $2^{\circ}$ Teste de Aptidão Física (TAF) anual, cada militar, por mais novo que fosse, já o teria realizado por, pelo menos, três vezes ${ }^{8}$.

O exame bioquímico foi realizado no laboratório da Diretoria de Pesquisa e Estudos de Pessoal (DPEP), em jejum de 12 horas, período em que poderia ingerir apenas água. Sempre que possível, foi evitada a administração de medicamentos nos três dias que antecederam o exame. Caso contrário, o fato seria registrado, assim como os medicamentos ingeridos.

Foram coletados dois tubos de ensaio de $4,5 \mathrm{ml}$ de sangue para as medidas bioquímicas. Dosou-se o nível sérico de glicemia, CT, LDL-c, HDL-c e TG. Para a análise bioquímica do colesterol total e frações, o sangue foi separado, por centrifugação, a $3000 \mathrm{rpm}$, durante 20 
minutos após coagulação e ficou em banho-maria de $37^{\circ}$, por 15 minutos. Para a análise da glicose, $20 \mu \mathrm{l}$ de soro receberam $2 \mathrm{ml}$ de reagente e permaneceram em banho-maria de $37^{\circ} \mathrm{C}$, por 10 minutos. As leituras foram realizadas em espectofotômetro com regulagem de 505 nanômetros. Para análise do nível de triglicerídeos, $10 \mu \mathrm{l}$ de soro receberam $1 \mathrm{ml}$ de reagente, após banho-maria de 10 minutos. A leitura foi realizada em espectofotômetro (500 nanômetros).

Considerou-se, como fatores de risco cardiovascular, os níveis séricos de: CT $\geq 240 \mathrm{mg}^{-\mathrm{dl}^{-1}}$, LDL-C $\geq 190 \mathrm{mg}^{-\mathrm{dl}^{-1}}$, HDL-c $<40 \mathrm{mg}^{-1 \mathrm{dl}^{-1}}$, glicemia em jejum $\geq 100 \mathrm{mg}^{\mathrm{dl}}{ }^{1}$ e TG $\geq 200 \mathrm{mg}^{\mathrm{ddl}}{ }^{-1}$.

Utilizou-se o teste de Shapiro-Wilk para verificar a distribuição dos dados e, após isto, optou-se por utilizar testes estatísticos não-paramétricos pelo fato de não haver encontrado normalidade na curva de distribuição. Para a análise de correlação, foi utilizado o teste de correlação de Spearman e, para a análise dos dados de maneira categorizada, o teste exato de Fisher (todos com significância de 0,05). Foi utilizado o software Stata 8.0 para Windows.

\section{RESULTADOS}

Os valores médios da amostra indicam que os níveis séricos de CT, HDL-c, LDL-c, TG e glicemia de jejum se encontram abaixo dos sugeridos como de risco pela literatura científica (TABELA 1).
Os dados encontrados sugerem que existe correlação inversa entre $\mathrm{VO}_{2 \max }$ e os níveis séricos de $\mathrm{CT}$ ( $\mathrm{r}=-0,490$; $p<0,05)$ e LDL-c $(r=-0,539 ; p<0,05)$, além de correlação direta com o HDL-c $(r=0,591 ; p<0,05)$, indicando que $o$ condicionamento cardiopulmonar pode atuar como fator de proteção para estes indicadores de DCV (GRÁFICOS 1 a 3). No entanto, não foram encontradas correlações entre o $\mathrm{VO}_{2 \max }$ e os níveis séricos de TG e a glicemia de jejum.

A aquisição de um $\mathrm{VO}_{2 \max }$ acima de $37,1 \mathrm{ml} \cdot \mathrm{kg}^{-1} \cdot \mathrm{min}^{-1}$ relacionou-se com a diminuição da prevalência de indivíduos com níveis séricos de risco de LDL-c (77,7\%x14,3\%; $p<0,05)$ e CT $(66,6 \% \times 12,5 \%$; $p<0,05)$, bem como com o aumento da prevalência de indivíduos com HDL-c acima de $40{\mathrm{mg} . \mathrm{dl}^{-1}}^{-1}(28,6 \% \times 68,3 \%$; $p<0,05)$ (GRÁFICO 4).

Com relação às características da atividade física realizada, a freqüência semanal de atividade física mostrou influência inversa na prevalência de sujeitos com IMC >25 $\mathrm{kg} \cdot \mathrm{m}^{-2}$, encontrando-se $50 \%$ de sujeitos, nesta faixa, entre aqueles que realizavam mais de 3 sessões semanais e $100 \%$, naqueles que realizavam no máximo 3 sessões $(p<0,05)$.

\section{DISCUSSÃO}

Ao se avaliar os efeitos do exercício físico sobre os diferentes fatores de risco de DCV, vários autores apresentam dados bastante semelhantes quanto ao

TABELA 1

CARACTERÍSTICAS DA AMOSTRA ( $\mathrm{N}=19)$.

\begin{tabular}{|c|c|c|c|c|}
\hline Variável & Média & $\mathrm{Dp}$ & Mínimo & Máximo \\
\hline Idade (anos) & 38,1 & 7,8 & 21,3 & 49,8 \\
\hline Massa Corporal (kg) & 89,3 & 7,1 & 72,7 & 99,2 \\
\hline Estatura $(\mathrm{cm})$ & 172,7 & 5,6 & 162,6 & 182,0 \\
\hline IMC $\left(\mathrm{kg} \cdot \mathrm{m}^{-2}\right)$ & 30,0 & 2,35 & 27,0 & 34,2 \\
\hline$\%$ Gordura & 24,9 & 4,2 & 17,5 & 31,3 \\
\hline Colesterol Total $\left(\mathrm{mg} \cdot \mathrm{dl}^{-1}\right)$ & 206 & 40,4 & 146,0 & 278,0 \\
\hline $\mathrm{HDL}-\mathrm{c}\left(\mathrm{mg} \cdot \mathrm{dl}^{-1}\right)$ & 44,1 & 9,3 & 34,0 & 64,0 \\
\hline LDL-c (mg.dl-1 $\left.{ }^{-1}\right)$ & 131,0 & 32,3 & 87,0 & 182,2 \\
\hline Triglicerídeos $\left(\mathrm{mg} \mathrm{dl}^{-1}\right)$ & 147,2 & 66,1 & 77,0 & 347,0 \\
\hline Glicemia $\left(\mathrm{mg} \mathrm{dl}{ }^{-1}\right)$ & 86,4 & 14,6 & 57,0 & 104,0 \\
\hline $\mathrm{VO}_{2 \max }\left(\mathrm{ml} \cdot \mathrm{kg}^{-1} \cdot \mathrm{min}^{-1}\right)$ & 43,2 & 8,8 & 31,2 & 62,4 \\
\hline
\end{tabular}


GRÁFICO 1

RELAÇÃO ENTRE VO ${ }_{2 M A X}$ E NÍVEIS SÉRICOS DE COLESTEROL TOTAL.

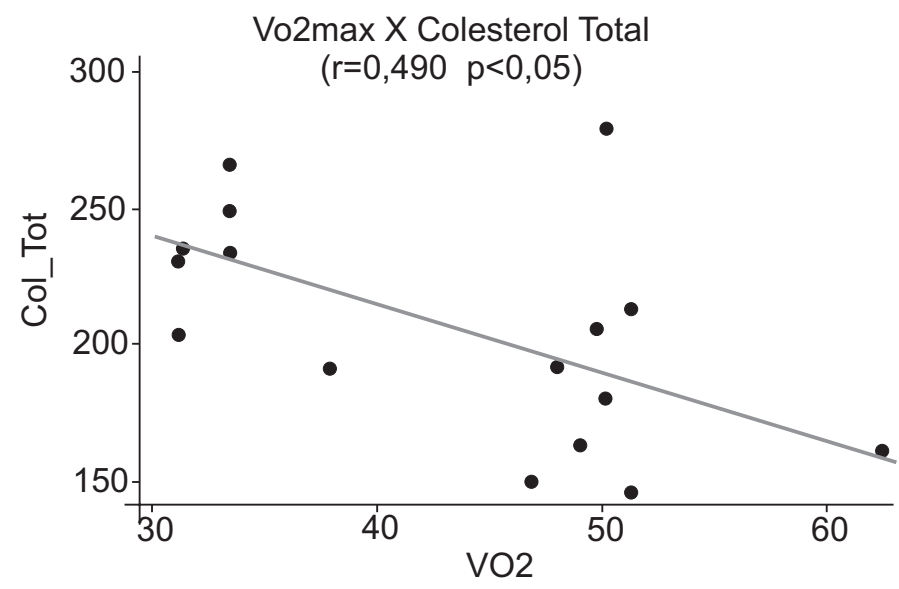

GRÁFICO 2

RELAÇÃO ENTRE VO ${ }_{2 M A X}$ E NÍVEIS

SÉRICOS DE LDL-COLESTEROL.

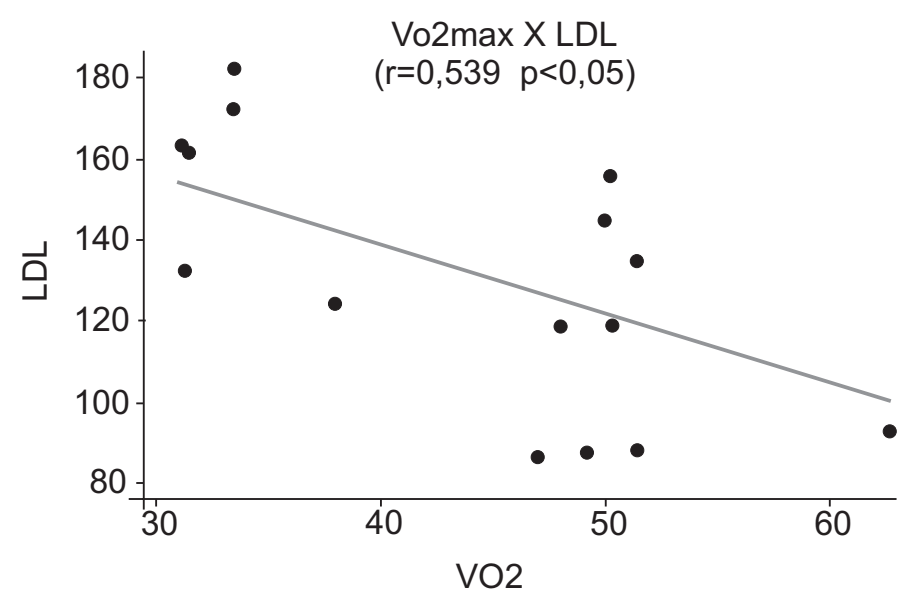

benefício dessa prática, tanto como coadjuvante terapêutico, como fator de profilaxia, sobretudo quando em associação com a perda de massa gorda e em programas dietéticos. A relação protetora do $\mathrm{VO}_{2 \max }$, encontrada nesse estudo, está de acordo com o que sugere a literatura científica $^{6,9-11}$, que afirma existir correlação direta do condicionamento cardiorrespiratório com os níveis de HDL-c e inversa, com os níveis de colesterol total e de LDL-C, assim como o percentual de gordura e o IMC.

Aparentemente, o tipo de sistema energético, predominante no exercício físico, apresenta influência limitada sobre os dados bioquímicos. Sabia et al. ${ }^{12}$ e

\section{GRÁFICO 3

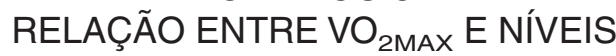 SÉRICOS DE HDL-COLESTEROL.}

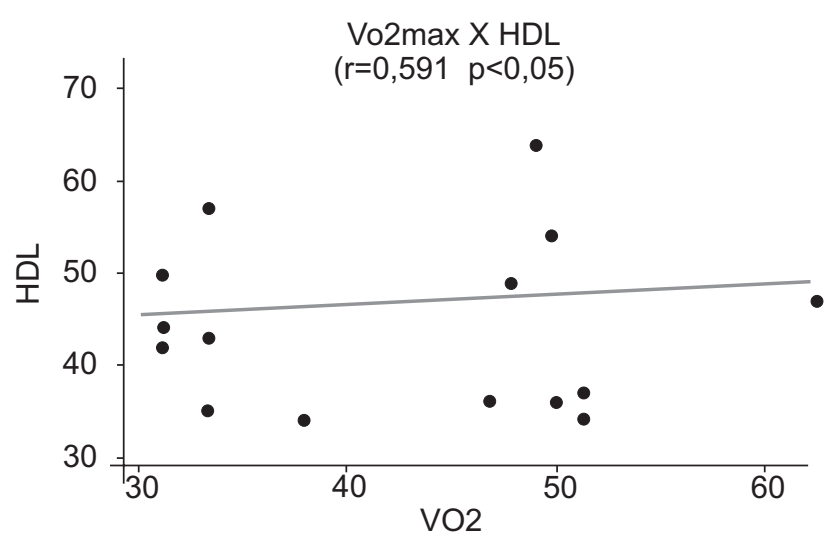

GRÁFICO 4

PREVALÊNCIA DE FATORES DE RISCO
PELO CONDICIONAMENTO
CARDIORRESPIRATÓRIO.

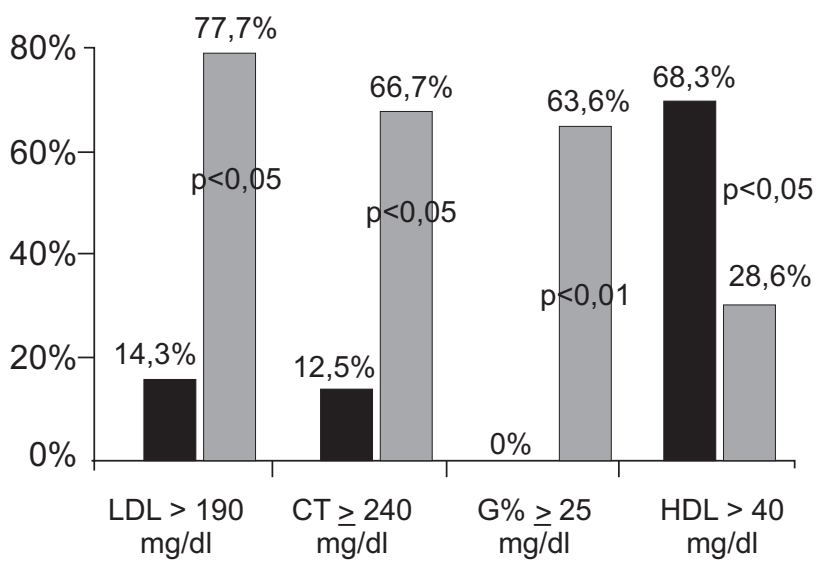

Bestetti \& Santos $^{13}$, sugerem que, em sujeitos com IMC elevado, os exercícios aeróbicos melhoraram de modo mais evidente o HDL-c, o LDL-c e o CT, em comparação com as atividades anaeróbicas, mais eficientes para o TG. Este estudo, também, corrobora os achados deste trabalho, considerando que os sujeitos do estudo realizavam, predominantemente, atividades aeróbicas.

No caso específico das alterações bioquímicas, os resultados, embora menos homogêneos quanto aos aspectos quantitativos das diferentes variáveis, também se mostraram significativos. Barbato et al. ${ }^{14}$ sugerem que 
um menor peso corporal, sob um regime de exercícios físicos e dieta, estabelece valores de redução nos níveis de CT e de LDL-c. Resultados semelhantes ${ }^{9,15}$, também, apontam para a influência do \%G com os níveis de LDL-c e glicemia de jejum, corroborando achados desse trabalho.

Os parâmetros de mensuração do condicionamento físico, assim como de sua influência no organismo, se mostraram qualitativamente adequados, mesmo em suas diferenças e peculiaridades. Anjos ${ }^{16}$ ressalta a validade do uso do IMC, sobretudo quanto ao aspecto da praticidade do método como um facilitador. Estudos de Lotufo ${ }^{17}$, do $\mathrm{NIH}^{18}$ (2001) e de Martinez ${ }^{9}$ sugerem que o IMC influencia a incidência de hipercolesterolemia, de maneira geral, e sofre influência das características da atividade física, o que está de acordo com os achados.

\section{CONCLUSÃO}

De maneira geral, sugere-se que se busque, como fatores de proteção cardiovascular, a manutenção do peso corporal em padrões adequados e o aumento do condicionamento cardiorrespiratório, por meio de atividades moderadas e intensas. É interessante o uso de instrumentos, como publicações em Noticiários do Exército e palestras nos Grandes Comandos, capazes de conscientizar os militares e o público em geral, da eficácia e da eficiência da adoção de hábitos de vida saudáveis para aquisição de uma qualidade de vida que permita realizar as tarefas laborais satisfatoriamente e, ainda, possibilite ao militar estar em condições de cumprir a atividade-fim para a qual de destina.

\section{REFERÊNCIAS BIBLIOGRÁFICAS}

1. Brasil. Ministério da Saúde, 2001. Informações hospitalares do DataSus. Disponível em: URL: <http://portal.saude.gov.br/ portal/aplicacoes/anuario2001/index.cfm> ( 08 fev. 2005).

2. Brasil. Ministério da Saúde, 1998. Divisão Nacional de Epidemiologia - Informe Epidemiológico do SUS. Brasília, DF: CENEA / FNS / MS; 1998.

3. Brasil. Ministério da Defesa. Anuário Estatístico do Exército. Brasília, DF: Estado-Maior do Exército; 2005.

4. Heath G, Hagberg J, Ehsani A, Holloszy J. A physiological comparison of young and older endurance athletes. JAP 1981;51:634-40.

5. Fletcher GF, Ballady G, Froelicher VF, Hartley H, Haskell WL, Pollock ML. A statement for healthcare professionals from the American Heart Association: exercise standards. Circulation 1992;86:340-4.

6. Laukkanen JA, Lakka TA, Rauramaa R, Kuhanen R, Venäläinen JM, Salonen R, et al. Cardiovascular fitness as a predictor of mortality in men. Arch Intern Med 2001;161:825-31.

7. Cooper KH. A means of assessing maximal oxigen intake. JAMA 1968;203:135-8.

8. Brasil. Diretriz para o TFM e sua Avaliação - Boletim do Exército 17. Brasília, DF: Estado-Maior do Exército; 1999.

9. Martinez EC. Fatores de risco de doencas ateroscleróticas coronarianas em militares da ativa do exército brasileiro com idade superior a 40 anos. Dissertação de Mestrado. Escola Nacional de Saúde Pública/Fiocruz. Março de 2004.

10. Kujala UM, Kaprio J, Sarna S, Koskenvuo M. Relationship of leisure-time physical activity and mortality: the Finnish twin cohort. JAMA 1998;279:440-4.

11. Blair SN, Kohl III HW, Paffenbarger Jr RS, Clark DG, Cooper GH, Gibbons LW. Physical fitness and all-cause mortality: a prospective study of health men and women. JAMA1989; 262:2395-401.

12. Sabia RV, Santos JE, Ribeiro RPP. Efeito da atividade física associada à orientação alimentar em adolescentes obesos: comparação entre o exercício aeróbio e anaeróbio. Rev Bras Med Esp 2004;10(5):349-55. 
13. Plaisance EP, Taylor JK, Alhassan S, Abebe A, Mestek ML, Grandjean PW. Cardiovascular fitness and vascular inflammatory markers after acute aerobic exercise. Int J Sport Nutr Exerc Metab 2007;17(2):152-62.

14. Barbato KBG, Martins RCV, Rodrigues MLG, Braga JU, Francischetti EA, Genelhu V. Efeitos da redução de peso superior a $5 \%$ nos perfis hemodinâmicos, metabólico e neuroendócrino de obesos grau I. Arq Bras Cardiol 2006;87(1):12-21.

15. Yamamoto A, Temba H, Horibe H, Mabuchi H, Saito Y, Matsuzawa Y, et al. Research Group on Serum Lipid Survey 1990 in Japan. Life style and cardiovascular risk factors in the Japanese population--from an epidemiological survey on serum lipid levels in Japan 1990 part 1: influence of life style and excess body weight on HDL-cholesterol and other lipid parameters in men. J Atheroscler Thromb 2003,10(3):165-75.

16. Anjos LA. Índice de massa corporal como indicador do estado nutricional de adultos: revisão de literatura. Rev Saude Publica 1992;26:431-6.

17. Lotufo PA. Mortalidade precoce por doença do coração no Brasil. Comparação com outros países. Arq Bras Cardiol 1998; 70:321-5.

18. NIH (National Institutes of Health). 2001. National Cholesterol Education Program. Adult Treatment Panel III Report. Disponível em: URL: <http://www.nhlbi.nih.gov/guidelines/cholesterol/atp3_rtp.htm> (20 dez. 2002).

\section{Endereço para correspondência:}

Eduardo Camillo Martinez Instituto de Pesquisa da Capacitação Física do Exército (IPCFEx) Av. João Luís Alves, s/no - Fortaleza de São João / Urca

Rio de Janeiro - RJ - Brasil

CEP 22291-090

Tel.: 2295-5340

e-mail: eduardocmartinez@gmail.com

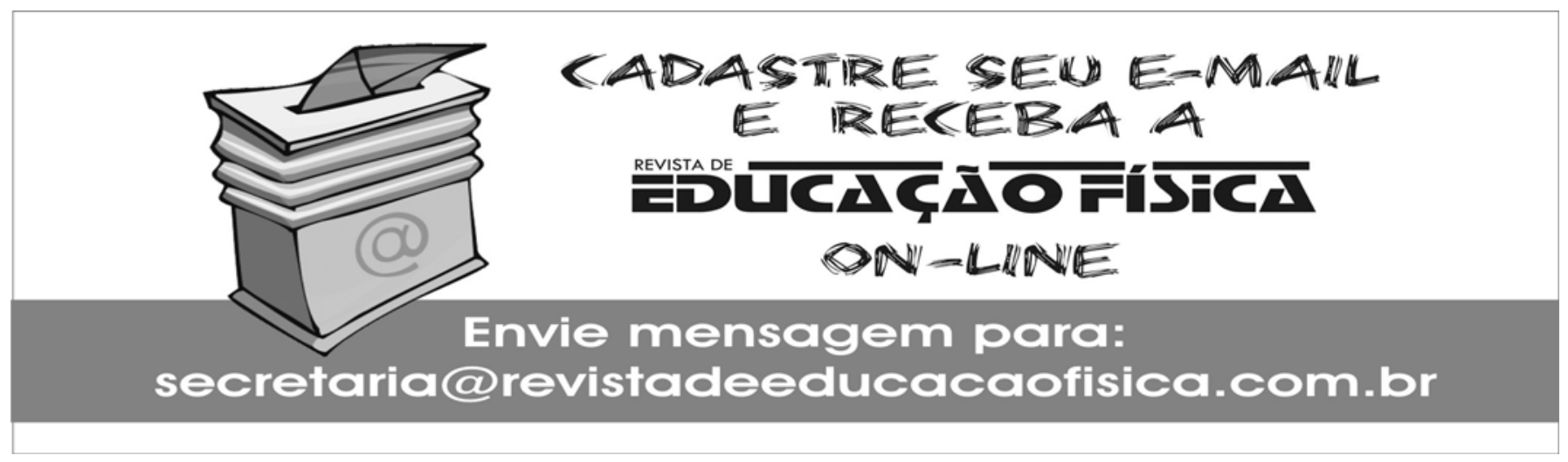

